\title{
Papers
}

\section{Used infant mattresses and sudden infant death syndrome in Scotland: case-control study}

\author{
David Tappin, Hazel Brooke, Russell Ecob, Angus Gibson
}

\begin{abstract}
Objective To examine the proposition that a used infant mattress is associated with an increased risk of sudden infant death syndrome.

Design Case-control study.

Setting Scotland (population 5.1 million, with about 53000 births a year).

Participants 131 infants who died of sudden infant death syndrome between 1 January 1996 and 31 May 2000 and 278 age, season, and obstetric unit matched control infants.

Main outcome measures Routine use of an infant mattress previously used by another child and place of last sleep.

Results Routine use of an infant mattress previously used by another child was significantly associated with an increased risk of sudden infant death syndrome (multivariate odds ratio 3.07, 95\% confidence interval 1.51 to 6.22$)$. Use of a used infant mattress for last sleep was also associated with increased risk (6.10, 2.31 to 16.12 ). The association was significantly stronger if the mattress was from another home (4.78, 2.08 to 11.0$)$ than if it was from the same home (1.64, 0.64 to 4.2 ).

Conclusion A valid significant association exists between use of a used infant mattress and an increased risk of sudden infant death syndrome, particularly if the mattress is from another home. Insufficient evidence is available to judge whether this relation is cause and effect.
\end{abstract}

\section{Introduction}

In 1997 we reported a study that suggested that babies sleeping on an infant mattress previously used by another child (used infant mattress) had an increased risk of sudden infant death syndrome. ${ }^{1}$ In this paper we report new case-control data from Scotland, gathered from 1 January 1996 to 31 May 2000 using a revised questionnaire. The aim of the study was to examine the null hypothesis that a used infant mattress is not associated with an increased risk of sudden infant death syndrome.

\section{Participants and methods}

The study was approved by ethics committees for all 15 health board areas in Scotland. The Registrar General for Scotland reported to us all infant deaths occurring after the seventh day of life to the end of the first year and provided the computerised maternity record. For sudden unexpected deaths, we were notified directly by the pathologist. A standard necropsy protocol with agreed diagnostic criteria was used to ensure consistent classification. ${ }^{2}$ We scrutinised all infant deaths for misclassification. Overall, 195 out of 751 postperinatal infant deaths were categorised as due to the sudden infant death syndrome between January 1996 and May 2000.

We identified babies born immediately before and after the index case in the same maternity unit to act as controls. Controls were therefore matched for age, season, and maternity unit. We made home visits to complete a questionnaire within 28 days of the index case's death to minimise differences in age related circumstances between cases and controls. Questionnaires were completed on 131 of 195 cases and 278 controls. We were unable to acquire data on 64 cases because a delay in notification by the pathologist made it impossible to visit within 28 days of the death. The characteristics of the cases with and without an interview were similar in terms of maternal age and deprivation category.

\section{Data collection}

The questionnaire provided core medical and social data as well as details of infant care practices. The difference from our previous study ${ }^{1}$ was that we collected data from control families about child care practices on the day and night before interview so that we could compare them with data on cases for the day and night of death (referred to as last sleep). We asked about cosleeping for last sleep, including where, for how long, and with whom.

Socioeconomic status was assessed by two sets of indicators. The first was an assessment of deprivation based on postcode of residence in seven categories in ascending order of deprivation (DEPCAT). ${ }^{3}$ The second was an individual assessment based on mother's marital status, mother living alone, mother and father currently employed, age mother and father left full time education, and whether the mother was in paid employment before the birth of the baby. We assessed exposure to smoking by determining use of cigarettes by the mother, father, and other household members.

\section{Editorial by Fleming et al \\ Scottish Cot Death Trust, Royal Hospital for Sick Children, Glasgow G3 8SJ \\ David Tappin \\ clinical senior lecturer \\ Hazel Brooke executive director \\ Russell Ecob research statistician Angus Gibson chairman \\ Correspondence to: D M Tappin, Paediatric Epidemiology and Community Health (PEACH) Unit, Department of Child Health, Royal Hospital for Sick \\ Children, Glasgow G3 8SJ goda11@udcf.gla. ac.uk}

bmj.com 2002;325:1007 
Exposure to a used infant mattress was assessed by asking parents about routine night and day sleeping place for both cases and controls and ascertaining the state of the mattress (new for this baby or, if not, how many other infants had used it and whether it came from another home). We also determined the last place of sleep for cases, whether day or night, and place of sleep for controls the day and night immediately before the interview.

\section{Analysis of data}

The full dataset included 131 cases and 278 controls (table). For the main analysis, we excluded babies who routinely slept in their parents bed at night (14 cases and 13 controls) as they did not sleep on an infant mattress. We used the random effects logistic regression procedure (xtlogit) in the software package Stata, ${ }^{4}$ which allows the inclusion of all the data, including those cases with no controls. We treated all variables that were significantly $(\mathrm{P}<0.05)$ related to sudden infant death syndrome on univariate analysis as potential confounding variables. The variables were entered sequentially in groups (socioeconomic, not modifiable, possibly modifiable) into the logistic regression of case versus control and removed singly in a stepwise manner starting with the least significant until all remaining variables were significant with a probability of $<0.10$. The next group of variables was then added and examined similarly. Quadratic functions were included for continuous and multicategorical variables and were retained if non-linear effects were present.

We established the model that best described the difference between cases and controls without the used infant mattress variables (table). This included: deprivation category, maternal age and its quadratic function, infant age and its quadratic function, parity (linear), admission to neonatal intensive care (yes/no), sharing a bed, couch, or chair during last sleep (yes/no), use of dummy during last sleep (no/a little), mother only smokes, both parents smoke, and laid prone to sleep (table). We then added the routine used infant mattress variable (yes/no) into the model. We also forced into the model the strongest other socioeconomic variable: mother not in paid employment before baby's birth. Further analyses were made of the excess risk of two or more mattress users versus one user, and used mattress from other home versus used mattress from same home.

We did two sensitivity analyses. In the first, we retained only cases occurring on a Sunday to Thursday $(\mathrm{n}=91)$ and their matched controls $(\mathrm{n}=205)$, as no designated last sleep for controls was on a Friday or Saturday (all interviews being carried out Monday to Friday). In the second we restricted cases to those found on infant mattresses who had not shared at any stage during their last sleep $(n=44)$ and controls who had slept on infant mattresses on their designated night and had not shared $(\mathrm{n}=205)$.

For dataset $\mathrm{B}$, using only cases $(\mathrm{n}=111)$ with one or more matched controls $(n=202)$, we built a multivariate model in the same way as in the main analysis but used conditional fixed effects logistic regression (clogit) to establish that the random effects method had not allowed bias. The model included deprivation category, maternal age, parity, intensive care, sharing, dummy, mother smokes, both parents smoke, laid prone, and laid on side. Routine use of a used mattress was then added to the model (table).

\section{Results}

The table shows the frequencies of all variables collected in the questionnaire. The main analysis showed a significant association between sudden infant death syndrome and routine use of a used infant mattress (odds ratio 3.07, 95\% confidence interval 1.93 to 5.06). When the variable mother not in paid employment before baby's birth was forced back into the multivariate model, the association remained unchanged $(3.04,1.49$ to 6.16$)$. The association remained when Friday/Saturday cases and controls were removed $(3.31,1.50$ to 7.34$)$ and when the dataset was restricted to those on an infant mattress throughout last sleep $(6.10,2.31$ to 16.10). A stronger association was seen for two or more previous users than for one, but this difference was not significant. With used infant mattress from the same home as the comparison group, an infant mattress from another home was significantly associated with sudden infant death syndrome (2.91, 1.02 to 8.33). A parallel multivariate conditional logistic regression with only matched cases and controls confirmed a significant association (9.59, 2.10 to 43.80$)$.

\section{Discussion}

This study tested the null hypothesis that a used infant mattress is not associated with an increased risk of the sudden infant death syndrome, based on new data collected in Scotland from January 1996 to May 2000. The first step is to assess if a valid statistical association exists - that is, that chance, bias, and confounding are unlikely alternative explanations. The second stage is to examine cause and effect.

\section{Role of chance, bias, and confounding}

As the focus of this study was on two issues (sharing a bed, couch, or chair during last sleep and use of a previously used infant mattress), a spurious finding of significance because of multiple statistical tests could not arise. We found that the null hypothesis of no association with a used infant mattress is rejected with a $\mathrm{P}$ value $<0.001$. A chance finding is therefore unlikely.

Bias is possible when the choice of cases and controls is affected by a systematic error. We could interview only 131 of 195 cases within 28 days because of delays in notification by pathologists. However, cases interviewed and not interviewed were not significantly different in terms of socioeconomic status measured by deprivation category or in terms of maternal age.

We used babies born just before and after the case in the same hospital as controls. However, a systematic difference in infant age for questions about last sleep (night before interview for controls, night of death for cases) was present (mean 18.5 days). The multivariate random effects model included a continuous linear variable for infant age and a quadratic function to cope with non-linearity, so it is unlikely that this source of bias explains the association. When cases where death occurred on a Friday and Saturday were excluded 
Univariate and multivariate random effects analysis without used mattress and with used mattress and conditional multivariate analysis with used mattress for deaths from sudden infant death syndrome and matched controls

\begin{tabular}{|c|c|c|c|c|c|c|c|c|c|c|c|c|c|c|}
\hline \multirow[b]{2}{*}{ Risk factor } & \multicolumn{2}{|c|}{$\begin{array}{c}\text { No (\%) of full } \\
\text { dataset }\end{array}$} & \multicolumn{2}{|c|}{$\begin{array}{c}\text { No (\%) of dataset } \\
A^{\star}\end{array}$} & \multicolumn{2}{|c|}{$\begin{array}{c}\text { No }(\%) \text { of dataset } \\
\text { B† }\end{array}$} & \multicolumn{2}{|c|}{$\begin{array}{l}\text { Dataset Aunivariate } \\
\text { analysis }\end{array}$} & \multicolumn{2}{|c|}{$\begin{array}{l}\text { Dataset A multivariate } \\
\text { random effects without } \\
\text { used mattress }\end{array}$} & \multicolumn{2}{|c|}{$\begin{array}{l}\text { Dataset A multivariate } \\
\text { random effects with } \\
\text { used mattress }\end{array}$} & \multicolumn{2}{|c|}{$\begin{array}{c}\text { Dataset B conditional } \\
\text { multivariatewith used } \\
\text { mattress }\end{array}$} \\
\hline & $\begin{array}{l}\text { Cases } \\
(\mathrm{n}=131)\end{array}$ & $\begin{array}{l}\text { Controls } \\
(\mathrm{n}=278)\end{array}$ & $\begin{array}{l}\text { Cases } \\
(\mathrm{n}=117)\end{array}$ & $\begin{array}{l}\text { Controls } \\
(\mathrm{n}=265)\end{array}$ & $\begin{array}{l}\text { Cases } \\
(\mathrm{n}=111)\end{array}$ & $\begin{array}{l}\text { Controls } \\
(\mathrm{n}=202)\end{array}$ & $\begin{array}{c}\text { OR } \\
(95 \% \mathrm{Cl})\end{array}$ & $\begin{array}{c}\mathrm{P} \\
\text { value }\end{array}$ & $\begin{array}{c}\text { OR } \\
(95 \% \mathrm{Cl})\end{array}$ & $\begin{array}{c}P \\
\text { value }\end{array}$ & $\begin{array}{c}\text { OR } \\
(95 \% \text { Cl) }\end{array}$ & $\begin{array}{c}\mathbf{P} \\
\text { value }\end{array}$ & $\begin{array}{c}\text { OR } \\
(95 \% \text { Cl) }\end{array}$ & $\begin{array}{c}P \\
\text { value }\end{array}$ \\
\hline \multicolumn{15}{|c|}{ Socioeconomic factors } \\
\hline $\begin{array}{l}\text { Mother } \\
\text { unmarried }\end{array}$ & $\begin{array}{c}91 / 131 \\
(69)\end{array}$ & $\begin{array}{c}113 / 278 \\
(41)\end{array}$ & $\begin{array}{c}82 / 117 \\
(70)\end{array}$ & $\begin{array}{c}108 / 265 \\
(41)\end{array}$ & $\begin{array}{c}79 / 111 \\
(71)\end{array}$ & $\begin{array}{c}78 / 202 \\
(39)\end{array}$ & $\begin{array}{c}3.41 \\
\text { (2.09 to 5.58) }\end{array}$ & $<0.001$ & & $>0.1$ & & & & \\
\hline $\begin{array}{l}\text { Mother living } \\
\text { alone }\end{array}$ & $\begin{array}{l}19 / 131 \\
(15)\end{array}$ & $\begin{array}{c}21 / 278 \\
(8)\end{array}$ & $\begin{array}{l}18 / 117 \\
(15)\end{array}$ & $\begin{array}{l}21 / 265 \\
(8)\end{array}$ & $\begin{array}{c}18 / 111 \\
(16)\end{array}$ & $\begin{array}{c}18 / 202 \\
(9)\end{array}$ & $\begin{array}{c}2.11 \\
\text { (1.08 to } 4.13)\end{array}$ & 0.029 & & $>0.1$ & & & & \\
\hline $\begin{array}{l}\text { Father } \\
\text { unemployed }\end{array}$ & $\begin{array}{c}55 / 124 \\
(44)\end{array}$ & $\begin{array}{c}56 / 255 \\
(22)\end{array}$ & $\begin{array}{c}49 / 111 \\
(44)\end{array}$ & $\begin{array}{c}50 / 243 \\
(21)\end{array}$ & $\begin{array}{c}46 / 105 \\
(44)\end{array}$ & $\begin{array}{c}37 / 186 \\
(20)\end{array}$ & $\begin{array}{c}3.05 \\
(1.87 \text { to } 4.96)\end{array}$ & $<0.001$ & & $>0.1$ & & & & \\
\hline $\begin{array}{l}\text { Mother } \\
\text { unemployed/ } \\
\text { housewife }\end{array}$ & $\begin{array}{l}108 / 129 \\
d /(84)\end{array}$ & $\begin{array}{c}9129 / 274 \\
(47)\end{array}$ & $\begin{array}{c}95 / 115 \\
(83)\end{array}$ & $\begin{array}{c}120 / 261 \\
(46)\end{array}$ & $\begin{array}{c}91 / 110 \\
(83)\end{array}$ & $\begin{array}{c}88 / 200 \\
(44)\end{array}$ & $\begin{array}{c}5.58 \\
\text { (3.25 to 9.58) }\end{array}$ & $<0.001$ & & $>0.1$ & & & & \\
\hline $\begin{array}{l}\text { Mother left } \\
\text { fulltime } \\
\text { education } \\
\text { before } 17\end{array}$ & $\begin{array}{c}93 / 127 \\
(73)\end{array}$ & $\begin{array}{c}148 / 276 \\
(54)\end{array}$ & $\begin{array}{c}84 / 113 \\
(74)\end{array}$ & $\begin{array}{c}140 / 264 \\
(53)\end{array}$ & $\begin{array}{c}80 / 108 \\
(74)\end{array}$ & $\begin{array}{c}110 / 202 \\
(54)\end{array}$ & $\begin{array}{c}2.57 \\
\text { (1.58 to 4.17) }\end{array}$ & $<0.001$ & & $>0.1$ & & & & \\
\hline $\begin{array}{l}\text { Father left } \\
\text { fulltime } \\
\text { education } \\
\text { before } 17 \\
\end{array}$ & $\begin{array}{c}95 / 131 \\
(73)\end{array}$ & $\begin{array}{c}146 / 277 \\
(53)\end{array}$ & $\begin{array}{c}86 / 117 \\
(74)\end{array}$ & $\begin{array}{c}142 / 26 \\
(4)\end{array}$ & $\begin{array}{c}84 / 111 \\
(76)\end{array}$ & $\begin{array}{c}107 / 201 \\
(53)\end{array}$ & $\begin{array}{c}2.38 \\
\text { (1.48 to } 3.84)\end{array}$ & $<0.001$ & & $>0.1$ & & & & \\
\hline \multicolumn{15}{|c|}{ Deprivation category3: } \\
\hline 1 & $\begin{array}{l}3 / 131 \\
(2)\end{array}$ & $\begin{array}{c}17 / 278 \\
(6)\end{array}$ & $\begin{array}{l}2 / 117 \\
\text { (2) }\end{array}$ & $\begin{array}{c}16 / 265 \\
(6)\end{array}$ & $\begin{array}{l}2 / 111 \\
(2)\end{array}$ & $\begin{array}{c}14 / 202 \\
(7)\end{array}$ & 1 & & 1 & & 1 & & 1 & \\
\hline 2 & $\begin{array}{l}4 / 131 \\
(3)\end{array}$ & $\begin{array}{c}27 / 278 \\
(10)\end{array}$ & $\begin{array}{l}2 / 117 \\
(2)\end{array}$ & $\begin{array}{c}27 / 265 \\
(10)\end{array}$ & $\begin{array}{l}2 / 111 \\
(2)\end{array}$ & $\begin{array}{c}19 / 202 \\
(9)\end{array}$ & $\begin{array}{c}0.59 \\
(0.05 \text { to } 6.71)\end{array}$ & 0.90 & & & & & & \\
\hline 3 & $\begin{array}{l}19 / 131 \\
(15)\end{array}$ & $\begin{array}{c}52 / 278 \\
(19)\end{array}$ & $\begin{array}{c}16 / 117 \\
(14)\end{array}$ & $\begin{array}{c}52 / 265 \\
(20)\end{array}$ & $\begin{array}{c}15 / 111 \\
(14)\end{array}$ & $\begin{array}{c}40 / 202 \\
(20)\end{array}$ & $\begin{array}{c}2.46 \\
\text { (0.46 to 17.33) }\end{array}$ & 0.20 & & & & & & \\
\hline 4 & $\begin{array}{c}30 / 131 \\
(23)\end{array}$ & $\begin{array}{c}64 / 278 \\
(23)\end{array}$ & $\begin{array}{c}28 / 117 \\
(24)\end{array}$ & $\begin{array}{c}60 / 265 \\
(23)\end{array}$ & $\begin{array}{c}26 / 111 \\
(23)\end{array}$ & $\begin{array}{l}40 / 202 \\
(20)\end{array}$ & $\begin{array}{c}3.73 \\
\text { (0.74 to 25.28) }\end{array}$ & 0.13 & & 0011 & 1.35 & & 2.32 & \\
\hline 5 & $\begin{array}{c}24 / 131 \\
(18)\end{array}$ & $\begin{array}{c}49 / 278 \\
(18)\end{array}$ & $\begin{array}{c}23 / 117 \\
(20)\end{array}$ & $\begin{array}{c}47 / 265 \\
(18)\end{array}$ & $\begin{array}{c}22 / 111 \\
(20)\end{array}$ & $\begin{array}{c}39 / 202 \\
(19)\end{array}$ & $\begin{array}{c}3.91 \\
(0.76 \text { to } 36.96) \\
\end{array}$ & 0.12 & (1.06 to 1.59 ) & 0.011 & (1.08 to 1.67$)$ & 0.007 & (1.35 to 4.01 ) & 0.002 \\
\hline 6 & $\begin{array}{c}21 / 131 \\
(16)\end{array}$ & $\begin{array}{c}41 / 278 \\
(15)\end{array}$ & $\begin{array}{c}19 / 117 \\
(16)\end{array}$ & $\begin{array}{c}38 / 265 \\
(14)\end{array}$ & $\begin{array}{c}17 / 111 \\
(15)\end{array}$ & $\begin{array}{c}30 / 202 \\
(15)\end{array}$ & $\begin{array}{c}4.00 \\
(0.75 \text { to } 28.10)\end{array}$ & 0.13 & & & & & & \\
\hline 7 & $\begin{array}{c}30 / 131 \\
(23)\end{array}$ & $\begin{array}{c}28 / 278 \\
(10)\end{array}$ & $\begin{array}{c}27 / 117 \\
(23)\end{array}$ & $\begin{array}{l}25 / 265 \\
(9)\end{array}$ & $\begin{array}{c}27 / 111 \\
(24)\end{array}$ & $\begin{array}{c}20 / 202 \\
(10)\end{array}$ & $\begin{array}{c}7.41 \\
\text { (1.40 to } 51.98)\end{array}$ & 0.012 & & & & & & \\
\hline $\begin{array}{l}\text { Mother not in } \\
\text { paid work } \\
\text { before birth }\end{array}$ & $\begin{array}{c}102 / 131 \\
(78)\end{array}$ & $\begin{array}{c}194 / 278 \\
(34)\end{array}$ & $\begin{array}{c}90 / 117 \\
(77)\end{array}$ & $\begin{array}{c}87 / 265 \\
(33)\end{array}$ & $\begin{array}{c}85 / 111 \\
(77)\end{array}$ & $\begin{array}{c}64 / 202 \\
(32)\end{array}$ & $\begin{array}{c}6.82 \\
(4.02 \text { to } 11.62)\end{array}$ & $<0.001$ & & $>0.1$ & & & & \\
\hline \multicolumn{15}{|c|}{ Not modifiable factors } \\
\hline Male sex & $\begin{array}{c}81 / 131 \\
(62)\end{array}$ & $\begin{array}{c}143 / 278 \\
(51)\end{array}$ & $\begin{array}{c}70 / 117 \\
(60)\end{array}$ & $\begin{array}{c}138 / 265 \\
(52)\end{array}$ & $\begin{array}{c}67 / 111 \\
(60)\end{array}$ & $\begin{array}{c}100 / 202 \\
(50)\end{array}$ & $\begin{array}{c}1.37 \\
(0.88 \text { to } 2.13)\end{array}$ & 0.16 & & $>0.1$ & & & & \\
\hline $\begin{array}{c}\text { Birth weight } \\
<2.5 \mathrm{~kg}\end{array}$ & $\begin{array}{c}29 / 130 \\
(22)\end{array}$ & $\begin{array}{c}18 / 278 \\
(6)\end{array}$ & $\begin{array}{c}26 / 116 \\
(22)\end{array}$ & $\begin{array}{c}16 / 265 \\
(6)\end{array}$ & $\begin{array}{c}26 / 110 \\
(24)\end{array}$ & $\begin{array}{c}13 / 202 \\
(6)\end{array}$ & $\begin{array}{c}4.50 \\
(2.30 \text { to } 8.77)\end{array}$ & $<0.001$ & & $>0.1$ & & & & \\
\hline $\begin{array}{l}\text { Gestation } \\
<37 \text { weeks }\end{array}$ & $\begin{array}{c}28 / 130 \\
(22)\end{array}$ & $\begin{array}{c}13 / 278 \\
(5)\end{array}$ & $\begin{array}{c}24 / 116 \\
(21)\end{array}$ & $\begin{array}{c}12 / 265 \\
(5)\end{array}$ & $\begin{array}{c}24 / 110 \\
(22)\end{array}$ & $\begin{array}{c}11 / 202 \\
(5)\end{array}$ & $\begin{array}{c}5.50 \\
\text { (2.64 to 11.45) }\end{array}$ & $<0.001$ & & $>0.1$ & & & & \\
\hline Multiple birth & $\begin{array}{c}10 / 131 \\
(8)\end{array}$ & $\begin{array}{c}6 / 278 \\
(2)\end{array}$ & $\begin{array}{c}10 / 117 \\
(9)\end{array}$ & $\begin{array}{c}5 / 265 \\
(2)\end{array}$ & $\begin{array}{c}10 / 111 \\
(9)\end{array}$ & $\begin{array}{l}2 / 202 \\
(1)\end{array}$ & $\begin{array}{c}4.86 \\
\text { (1.62 to } 14.55) \\
\end{array}$ & 0.005 & & $>0.1$ & & & & \\
\hline $\begin{array}{l}\text { Mean }(\mathrm{SD}) \\
\text { maternal } \\
\text { age (years) }\end{array}$ & $\begin{array}{l}25.1 \\
(5.8)\end{array}$ & $\begin{array}{l}28.9 \\
(5.7)\end{array}$ & $\begin{array}{l}25.1 \\
(5.8)\end{array}$ & $\begin{array}{l}28.9 \\
(5.7)\end{array}$ & $\begin{array}{l}25.2 \\
(5.7)\end{array}$ & $\begin{array}{l}29.0 \\
(5.6)\end{array}$ & $\begin{array}{c}0.89 \\
(0.85 \text { to } 0.93)\end{array}$ & $<0.001$ & $\begin{array}{c}0.84 \\
(0.79 \text { to } 0.90)\end{array}$ & $<0.001$ & $\begin{array}{c}0.84 \\
(0.78 \text { to } 0.90)\end{array}$ & $<0.001$ & $\begin{array}{c}0.86 \\
(0.77 \text { to } 0.96)\end{array}$ & 0.006 \\
\hline $\begin{array}{l}\text { Quadratic } \\
\text { function } \\
\text { maternal age }\end{array}$ & & & & & & & $\begin{array}{c}1.00 \\
(1.00 \text { to } 1.01)\end{array}$ & 0.17 & $\begin{array}{c}1.01 \\
\text { (1.00 to } 1.02)\end{array}$ & 0.051 & $\begin{array}{c}1.01 \\
\text { (1.00 to } 1.02)\end{array}$ & 0.018 & & \\
\hline \multicolumn{15}{|l|}{ Parity: } \\
\hline 1 & $\begin{array}{c}40 / 131 \\
(31)\end{array}$ & $\begin{array}{c}129 / 278 \\
(46)\end{array}$ & $\begin{array}{c}34 / 117 \\
(29)\end{array}$ & $\begin{array}{c}123 / 265 \\
(46)\end{array}$ & $\begin{array}{c}32 / 111 \\
(29)\end{array}$ & $\begin{array}{c}91 / 202 \\
(45)\end{array}$ & 1 & & 1 & & 1 & & 1 & \\
\hline 2 & $\begin{array}{c}41 / 131 \\
(31)\end{array}$ & $\begin{array}{c}96 / 278 \\
(35)\end{array}$ & $\begin{array}{c}39 / 117 \\
(33)\end{array}$ & $\begin{array}{c}93 / 265 \\
(35)\end{array}$ & $\begin{array}{c}37 / 111 \\
(33)\end{array}$ & $\begin{array}{c}73 / 202 \\
(36)\end{array}$ & $\begin{array}{c}1.52 \\
(0.86 \text { to } 2.68)\end{array}$ & 0.16 & & & & & & \\
\hline 3 & $\begin{array}{c}29 / 131 \\
(22)\end{array}$ & $\begin{array}{c}37 / 278 \\
(13)\end{array}$ & $\begin{array}{c}26 / 117 \\
(22)\end{array}$ & $\begin{array}{c}35 / 265 \\
(13)\end{array}$ & $\begin{array}{c}26 / 111 \\
(23)\end{array}$ & $\begin{array}{c}26 / 202 \\
(13)\end{array}$ & $\begin{array}{c}2.69 \\
\text { (1.36 to 5.32) }\end{array}$ & 0.003 & $\begin{array}{c}2.35 \\
\text { (1.69 to } 3.25)\end{array}$ & $<0.001$ & $\begin{array}{c}1.92 \\
\text { (1.34 to } 2.75)\end{array}$ & $<0.001$ & $\begin{array}{c}1.53 \\
\text { (0.86 to 2.74) }\end{array}$ & 0.15 \\
\hline$>3$ & $\begin{array}{c}21 / 131 \\
(16)\end{array}$ & $\begin{array}{c}16 / 278 \\
(6)\end{array}$ & $\begin{array}{c}18 / 117 \\
(15)\end{array}$ & $\begin{array}{l}14 / 265 \\
(5)\end{array}$ & $\begin{array}{c}16 / 111 \\
(14)\end{array}$ & $\begin{array}{c}12 / 202 \\
(6)\end{array}$ & $\begin{array}{c}4.65 \\
\text { (1.96 to 11.12) }\end{array}$ & $<0.001$ & & & & & & \\
\hline $\begin{array}{l}\text { Admitted } \\
\quad \text { neonatal } \\
\text { intensive } \\
\text { care }\end{array}$ & $\begin{array}{c}21 / 131 \\
(16)\end{array}$ & $\begin{array}{c}10 / 278 \\
(4)\end{array}$ & $\begin{array}{c}18 / 115 \\
(16)\end{array}$ & $\begin{array}{l}9 / 265 \\
(3)\end{array}$ & $\begin{array}{c}18 / 109 \\
(17)\end{array}$ & $\begin{array}{l}9 / 202 \\
(4)\end{array}$ & $\begin{array}{c}5.28 \\
(2.15 \text { to } 13.20)\end{array}$ & $<0.001$ & $\begin{array}{c}3.67 \\
(1.09 \text { to } 12.34)\end{array}$ & 0.036 & $\begin{array}{c}4.11 \\
\text { (1.12 to 14.99) }\end{array}$ & 0.032 & $\begin{array}{c}5.46 \\
\text { (1.01 to } 29.45)\end{array}$ & 0.048 \\
\hline $\begin{array}{l}\text { Mean infant } \\
\text { age (days) } \\
\text { (death/ } \\
\text { designated) } \\
\text { mean (SD) }\end{array}$ & $\begin{array}{c}94.3 \\
(67.1)\end{array}$ & $\begin{array}{l}114.8 \\
(65.7)\end{array}$ & $\begin{array}{l}95.3 \\
(66.5)\end{array}$ & $\begin{array}{l}113.8 \\
(66.0)\end{array}$ & $\begin{array}{c}94.4 \\
(66.7)\end{array}$ & $\begin{array}{l}113.9 \\
(67.3)\end{array}$ & $\begin{array}{c}1.00 \\
(0.99 \text { to } 1.00)\end{array}$ & 0.013 & $\begin{array}{c}0.99 \\
(0.99 \text { to } 1.00)\end{array}$ & 0.022 & $\begin{array}{c}0.99 \\
(0.99 \text { to } 1.00)\end{array}$ & 0.079 & & \\
\hline $\begin{array}{l}\text { Quadratic } \\
\text { function } \\
\text { infant age }\end{array}$ & & & & & & & $\begin{array}{c}1.00 \\
(1.00 \text { to } 1.00)\end{array}$ & 0.84 & $\begin{array}{c}1.00 \\
\text { (1.00 to } 1.00)\end{array}$ & 0.21 & $\begin{array}{c}1.00 \\
(1.00 \text { to } 1.00)\end{array}$ & 0.51 & & \\
\hline
\end{tabular}


Univariate and multivariate random effects analysis without used mattress and with used mattress and conditional multivariate analysis with used mattress for deaths from sudden infant death syndrome and matched controls-Continued from previous page

\begin{tabular}{|c|c|c|c|c|c|c|c|c|c|c|c|c|c|c|}
\hline \multirow[b]{2}{*}{ Risk factor } & \multicolumn{2}{|c|}{$\begin{array}{l}\text { No (\%) of full } \\
\text { dataset }\end{array}$} & \multicolumn{2}{|c|}{$\begin{array}{c}\text { No (\%) of dataset } \\
A^{\star}\end{array}$} & \multicolumn{2}{|c|}{$\begin{array}{c}\text { No (\%) of dataset } \\
\text { B十 }\end{array}$} & \multicolumn{2}{|c|}{$\begin{array}{l}\text { Dataset Aunivariate } \\
\text { analysis }\end{array}$} & \multicolumn{2}{|c|}{$\begin{array}{l}\text { Dataset A multivariate } \\
\text { random effects without } \\
\text { used mattress }\end{array}$} & \multicolumn{2}{|c|}{$\begin{array}{l}\text { Dataset A multivariate } \\
\text { random effects with } \\
\text { used mattress }\end{array}$} & \multicolumn{2}{|c|}{$\begin{array}{l}\text { Dataset B conditional } \\
\text { multivariatewith used } \\
\text { mattress }\end{array}$} \\
\hline & $\begin{array}{c}\text { Cases } \\
(\mathrm{n}=131)\end{array}$ & $\begin{array}{l}\text { Controls } \\
(\mathrm{n}=278)\end{array}$ & $\begin{array}{l}\text { Cases } \\
(\mathrm{n}=117)\end{array}$ & $\begin{array}{l}\text { Controls } \\
(\mathrm{n}=265)\end{array}$ & $\begin{array}{c}\text { Cases } \\
(\mathrm{n}=111)\end{array}$ & $\begin{array}{l}\text { Controls } \\
(\mathrm{n}=202)\end{array}$ & $\begin{array}{c}\text { OR } \\
(95 \% \mathrm{Cl})\end{array}$ & $\begin{array}{c}\mathbf{P} \\
\text { value }\end{array}$ & $\begin{array}{c}\text { OR } \\
(95 \% \mathrm{Cl})\end{array}$ & $\begin{array}{c}P \\
\text { value }\end{array}$ & $\begin{array}{c}\text { OR } \\
(95 \% \mathrm{Cl})\end{array}$ & $\begin{array}{c}P \\
\text { value }\end{array}$ & $\begin{array}{c}\text { OR } \\
(95 \% \mathrm{Cl})\end{array}$ & $\begin{array}{c}P \\
\text { value }\end{array}$ \\
\hline \multicolumn{15}{|c|}{ Possibly modifiable factors } \\
\hline $\begin{array}{l}\text { Ever found } \\
\text { under } \\
\text { bedclothes }\end{array}$ & $\begin{array}{c}23 / 128 \\
(18)\end{array}$ & $\begin{array}{c}74 / 277 \\
(27)\end{array}$ & $\begin{array}{c}20 / 114 \\
(18)\end{array}$ & $\begin{array}{c}69 / 264 \\
(26)\end{array}$ & $\begin{array}{c}19 / 108 \\
(18)\end{array}$ & $\begin{array}{c}48 / 202 \\
(24)\end{array}$ & $\begin{array}{c}0.60 \\
(0.35 \text { to } 1.05)\end{array}$ & 0.073 & & $>0.1$ & & & & \\
\hline $\begin{array}{l}\text { Cot made up } \\
\text { from } \\
\text { bottom }\end{array}$ & $\begin{array}{c}23 / 61 \\
(38)\end{array}$ & $\begin{array}{c}106 / 180 \\
(59)\end{array}$ & $\begin{array}{c}20 / 58 \\
(34)\end{array}$ & $\begin{array}{c}105 / 178 \\
(59)\end{array}$ & $\begin{array}{c}18 / 53 \\
(34)\end{array}$ & $\begin{array}{c}75 / 133 \\
(56)\end{array}$ & $\begin{array}{c}0.37 \\
\text { (0.19 to } 0.71)\end{array}$ & 0.002 & & $>0.1$ & & & & \\
\hline \multicolumn{15}{|c|}{ Feeding at death and at interview for controls: } \\
\hline Breast only & $\begin{array}{c}15 / 131 \\
(11)\end{array}$ & $\begin{array}{c}51 / 278 \\
(18)\end{array}$ & $\begin{array}{c}10 / 117 \\
(9)\end{array}$ & $\begin{array}{c}48 / 265 \\
(18)\end{array}$ & $\begin{array}{c}10 / 111 \\
(9)\end{array}$ & $\begin{array}{c}37 / 202 \\
(18)\end{array}$ & 1 & & & & & & & \\
\hline $\begin{array}{l}\text { Breast and } \\
\text { formula }\end{array}$ & $\begin{array}{c}15 / 131 \\
(11)\end{array}$ & $\begin{array}{c}33 / 278 \\
(12)\end{array}$ & $\begin{array}{c}14 / 117 \\
(12)\end{array}$ & $\begin{array}{c}32 / 265 \\
(12)\end{array}$ & $\begin{array}{c}13 / 111 \\
(12)\end{array}$ & $\begin{array}{c}24 / 202 \\
(12)\end{array}$ & $\begin{array}{c}2.10 \\
(0.83 \text { to } 5.30)\end{array}$ & 0.12 & & & & & & \\
\hline $\begin{array}{l}\text { Formula } \\
\text { only }\end{array}$ & $\begin{array}{c}101 / 131 \\
(77)\end{array}$ & $\begin{array}{c}194 / 278 \\
(70)\end{array}$ & $\begin{array}{c}93 / 117 \\
(79)\end{array}$ & $\begin{array}{c}185 / 265 \\
(70)\end{array}$ & $\begin{array}{c}88 / 111 \\
(79)\end{array}$ & $\begin{array}{c}141 / 202 \\
(70)\end{array}$ & $\begin{array}{c}2.41 \\
\text { (1.17 to 4.98) } \\
\end{array}$ & 0.017 & & $>0.1$ & & & & \\
\hline $\begin{array}{l}\text { Breastfed for } \\
\text { more than } \\
1 \text { week } \\
\end{array}$ & $\begin{array}{c}23 / 106 \\
(22)\end{array}$ & $\begin{array}{c}78 / 202 \\
(39)\end{array}$ & $\begin{array}{c}20 / 97 \\
(21)\end{array}$ & $\begin{array}{c}75 / 193 \\
(39)\end{array}$ & $\begin{array}{l}17 / 91 \\
(19)\end{array}$ & $\begin{array}{c}56 / 148 \\
(38)\end{array}$ & $\begin{array}{c}0.41 \\
\text { (0.22 to } 0.75)\end{array}$ & 0.003 & & $>0.1$ & & & & \\
\hline $\begin{array}{l}\text { Infant in own } \\
\text { bedroom } \\
\text { most of } \\
\text { last sleep }\end{array}$ & $\begin{array}{c}10 / 129 \\
(8)\end{array}$ & $\begin{array}{c}45 / 278 \\
(16)\end{array}$ & $\begin{array}{c}10 / 115 \\
(9)\end{array}$ & $\begin{array}{c}45 / 265 \\
(17)\end{array}$ & $\begin{array}{c}10 / 109 \\
(9)\end{array}$ & $\begin{array}{c}44 / 202 \\
(22)\end{array}$ & $\begin{array}{c}0.51 \\
\text { (0.23 to 1.11) }\end{array}$ & 0.10 & & & & & & \\
\hline \multicolumn{15}{|c|}{ Sharing bed, couch, or chair during last sleep: } \\
\hline No & $\begin{array}{c}65 / 131 \\
(50)\end{array}$ & $\begin{array}{c}223 / 278 \\
(80) \\
\end{array}$ & $\begin{array}{c}64 / 117 \\
(55)\end{array}$ & $\begin{array}{c}221 / 265 \\
(83) \\
\end{array}$ & $\begin{array}{c}60 / 111 \\
(54)\end{array}$ & $\begin{array}{c}165 / 202 \\
(82) \\
\end{array}$ & 1 & & 1 & & 1 & & 1 & \\
\hline 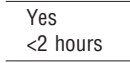 & $\begin{array}{c}8 / 131 \\
(6)\end{array}$ & $\begin{array}{c}15 / 278 \\
(5)\end{array}$ & $\begin{array}{c}8 / 117 \\
(7)\end{array}$ & $\begin{array}{c}15 / 265 \\
(6)\end{array}$ & $\begin{array}{c}8 / 111 \\
(7)\end{array}$ & $\begin{array}{c}13 / 202 \\
(6)\end{array}$ & $\begin{array}{c}1.84 \\
\text { (0.75 to 4.54) }\end{array}$ & 0.19 & & & & & & \\
\hline $\begin{array}{l}\text { Yes } \\
2-5 \text { hours }\end{array}$ & $\begin{array}{c}28 / 131 \\
(21) \\
\end{array}$ & $\begin{array}{c}15 / 278 \\
(5)\end{array}$ & $\begin{array}{c}25 / 117 \\
(21)\end{array}$ & $\begin{array}{c}15 / 265 \\
(6)\end{array}$ & $\begin{array}{c}23 / 111 \\
(21)\end{array}$ & $\begin{array}{c}12 / 202 \\
(6)\end{array}$ & $\begin{array}{c}5.76 \\
\text { (2.86 to } 11.57) \\
\end{array}$ & $<0.001$ & $\begin{array}{c}3.36 \\
(1.67 \text { to } 6.73)\end{array}$ & 0.001 & $\begin{array}{c}3.62 \\
\text { (1.69 to } 7.77)\end{array}$ & 0.001 & $\begin{array}{c}15.69 \\
(1.67 \text { to } 22.58)\end{array}$ & 0.006 \\
\hline $\begin{array}{l}\text { Yes } \\
>5 \text { hours }\end{array}$ & $\begin{array}{c}30 / 131 \\
(23)\end{array}$ & $\begin{array}{c}25 / 278 \\
(9)\end{array}$ & $\begin{array}{c}20 / 117 \\
(17)\end{array}$ & $\begin{array}{c}14 / 265 \\
(5)\end{array}$ & $\begin{array}{c}20 / 111 \\
(18)\end{array}$ & $\begin{array}{c}12 / 202 \\
(6)\end{array}$ & $\begin{array}{c}4.93 \\
(2.36 \text { to } 10.31) \\
\end{array}$ & $<0.001$ & & & & & & \\
\hline \multicolumn{15}{|c|}{ Use of dummy during last sleep: } \\
\hline None & $\begin{array}{c}78 / 130 \\
(60)\end{array}$ & $\begin{array}{c}140 / 278 \\
(50)\end{array}$ & $\begin{array}{c}69 / 116 \\
(59)\end{array}$ & $\begin{array}{c}132 / 265 \\
(50)\end{array}$ & $\begin{array}{c}67 / 110 \\
(61)\end{array}$ & $\begin{array}{c}103 / 202 \\
(51)\end{array}$ & 1 & & 1 & & 1 & & 1 & \\
\hline A little & $\begin{array}{c}30 / 130 \\
(23)\end{array}$ & $\begin{array}{c}97 / 278 \\
(35)\end{array}$ & $\begin{array}{c}27 / 116 \\
(23)\end{array}$ & $\begin{array}{c}94 / 265 \\
(35)\end{array}$ & $\begin{array}{c}23 / 110 \\
(21)\end{array}$ & $\begin{array}{c}66 / 202 \\
(33)\end{array}$ & $\begin{array}{c}0.55 \\
(0.32 \text { to } 0.95)\end{array}$ & 0.03 & $\begin{array}{c}0.65 \\
(0.42 \text { to } 1.01)\end{array}$ & 0.056 & $\begin{array}{c}0.72 \\
\text { (0.44 to } 1.17) \\
\end{array}$ & 0.19 & $\begin{array}{c}0.59 \\
(0.30 \text { to } 1.17) \\
\end{array}$ & 0.13 \\
\hline A lot & $\begin{array}{c}22 / 130 \\
(17)\end{array}$ & $\begin{array}{c}41 / 278 \\
(15)\end{array}$ & $\begin{array}{c}20 / 116 \\
(17)\end{array}$ & $\begin{array}{c}39 / 265 \\
(15)\end{array}$ & $\begin{array}{c}20 / 110 \\
(18)\end{array}$ & $\begin{array}{c}33 / 202 \\
(16)\end{array}$ & $\begin{array}{c}0.91 \\
(0.47 \text { to } 1.76)\end{array}$ & 0.90 & & & & & & \\
\hline \multicolumn{15}{|c|}{ Exposure to smoking: } \\
\hline $\begin{array}{l}\text { Neither } \\
\text { parent } \\
\text { smokes }\end{array}$ & $\begin{array}{c}17 / 121 \\
(14)\end{array}$ & $\begin{array}{c}145 / 268 \\
(54)\end{array}$ & $\begin{array}{c}15 / 109 \\
(14)\end{array}$ & $\begin{array}{c}141 / 256 \\
(55)\end{array}$ & $\begin{array}{c}12 / 103 \\
(12)\end{array}$ & $\begin{array}{c}109 / 195 \\
(56)\end{array}$ & 1 & & 1 & & 1 & & 1 & \\
\hline $\begin{array}{l}\text { Father } \\
\text { smokes } \\
\text { only }\end{array}$ & $\begin{array}{c}12 / 121 \\
(10)\end{array}$ & $\begin{array}{c}39 / 268 \\
(15)\end{array}$ & $\begin{array}{c}11 / 109 \\
(10)\end{array}$ & $\begin{array}{c}36 / 256 \\
(14)\end{array}$ & $\begin{array}{c}11 / 103 \\
(11)\end{array}$ & $\begin{array}{c}28 / 195 \\
(14)\end{array}$ & $\begin{array}{c}2.87 \\
\text { (1.12 to } 7.35)\end{array}$ & 0.026 & & $>0.1$ & & & & \\
\hline $\begin{array}{l}\text { Mother } \\
\text { smokes } \\
\text { only }\end{array}$ & $\begin{array}{c}24 / 121 \\
(20)\end{array}$ & $\begin{array}{c}35 / 268 \\
(13)\end{array}$ & $\begin{array}{c}22 / 109 \\
(20)\end{array}$ & $\begin{array}{c}33 / 256 \\
(13)\end{array}$ & $\begin{array}{c}22 / 103 \\
(21)\end{array}$ & $\begin{array}{c}23 / 195 \\
(12)\end{array}$ & $\begin{array}{c}6.27 \\
\text { (2.76 to } 14.36)\end{array}$ & $<0.001$ & $\begin{array}{c}4.39 \\
\text { (1.90 to } 10.14)\end{array}$ & 0.001 & $\begin{array}{c}4.59 \\
\text { (1.84 to } 11.45 \text { ) }\end{array}$ & 0.001 & $\begin{array}{c}26.78 \\
(3.99 \text { to } 179.4)\end{array}$ & 0.001 \\
\hline $\begin{array}{l}\text { Both } \\
\text { smoke }\end{array}$ & $\begin{array}{c}68 / 121 \\
(56)\end{array}$ & $\begin{array}{c}49 / 268 \\
(18)\end{array}$ & $\begin{array}{c}61 / 109 \\
(56)\end{array}$ & $\begin{array}{c}46 / 256 \\
(18)\end{array}$ & $\begin{array}{c}58 / 103 \\
(56)\end{array}$ & $\begin{array}{c}35 / 195 \\
(18)\end{array}$ & $\begin{array}{c}12.47 \\
\text { (6.19 to } 25.42) \\
\end{array}$ & $<0.001$ & $\begin{array}{c}6.46 \\
\text { (3.17 to 13.17) }\end{array}$ & $<0.001$ & $\begin{array}{c}5.79 \\
\text { (2.71 to } 12.38) \\
\end{array}$ & $<0.001$ & $\begin{array}{c}15.69 \\
(3.11 \text { to } 79.07) \\
\end{array}$ & 0.001 \\
\hline $\begin{array}{l}\text { Others } \\
\text { smoking } \\
\text { in the } \\
\text { household }\end{array}$ & $\begin{array}{c}11 / 130 \\
(8)\end{array}$ & $\begin{array}{c}20 / 278 \\
(7)\end{array}$ & $\begin{array}{l}9 / 116 \\
(8)\end{array}$ & $\begin{array}{c}18 / 265 \\
(7)\end{array}$ & $\begin{array}{c}8 / 110 \\
(7)\end{array}$ & $\begin{array}{c}14 / 202 \\
(7)\end{array}$ & $\begin{array}{c}1.15 \\
(0.46 \text { to } 2.82)\end{array}$ & 0.90 & & & & & & \\
\hline \multicolumn{15}{|c|}{ Position placed to sleep: } \\
\hline Supine & $\begin{array}{c}73 / 129 \\
(57) \\
\end{array}$ & $\begin{array}{c}216 / 278 \\
(78)\end{array}$ & $\begin{array}{c}64 / 115 \\
(56)\end{array}$ & $\begin{array}{c}206 / 265 \\
(78) \\
\end{array}$ & $\begin{array}{c}62 / 109 \\
(57)\end{array}$ & $\begin{array}{c}160 / 202 \\
(79)\end{array}$ & 1 & & 1 & & 1 & & 1 & \\
\hline Side & $\begin{array}{c}47 / 129 \\
(36)\end{array}$ & $\begin{array}{c}56 / 278 \\
(20)\end{array}$ & $\begin{array}{c}42 / 115 \\
(37)\end{array}$ & $\begin{array}{c}54 / 265 \\
(20)\end{array}$ & $\begin{array}{c}40 / 109 \\
(37)\end{array}$ & $\begin{array}{c}38 / 202 \\
(19)\end{array}$ & $\begin{array}{c}2.50 \\
\text { (1.53 to 4.09) }\end{array}$ & $<0.001$ & & $>0.1$ & & & $\begin{array}{c}2.81 \\
\text { (0.89 to } 8.83) \\
\end{array}$ & 0.077 \\
\hline Prone & $\begin{array}{c}9 / 129 \\
(7) \\
\end{array}$ & $\begin{array}{c}6 / 278 \\
(2) \\
\end{array}$ & $\begin{array}{c}9 / 115 \\
(8)\end{array}$ & $\begin{array}{c}5 / 265 \\
(2) \\
\end{array}$ & $\begin{array}{c}7 / 109 \\
(6)\end{array}$ & $\begin{array}{c}/ 202 \\
(2) \\
\end{array}$ & $\begin{array}{c}5.79 \\
\text { (1.87 to } 17.91) \\
\end{array}$ & 0.002 & $\begin{array}{c}4.54 \\
\text { (0.92 to 22.38) }\end{array}$ & 0.063 & $\begin{array}{c}3.33 \\
(0.50 \text { to } 22.36) \\
\end{array}$ & 0.22 & $\begin{array}{c}8.66 \\
(0.42 \text { to } 180.18) \\
\end{array}$ & 0.16 \\
\hline
\end{tabular}

because no designated last sleep for controls was on a Friday or Saturday, the association remained. Finally, a parallel conditional logistic regression using only cases with matched controls, and so controlling for any possible bias due to systematic differences in the cases for which controls did or did not exist, showed the same association. Some possible sources of bias to explain the observed association have been ruled out.
Confounding from socioeconomic status is the most obvious possibility for an association between sudden infant death syndrome and use of a used infant mattress. Deprivation category, which we derived from information not obtained by questionnaire, was the most resilient socioeconomic variable in the multivariate models. As this was the only socioeconomic variable that remained before introduction of used 
Univariate and multivariate random effects analysis without used mattress and with used mattress and conditional multivariate analysis with used mattress for deaths from sudden infant death syndrome and matched controls-Continued from previous page

\begin{tabular}{|c|c|c|c|c|c|c|c|c|c|c|c|c|c|}
\hline \multirow[b]{2}{*}{ Risk factor } & $\begin{array}{c}\text { No }(\%) \text { of full } \\
\text { dataset }\end{array}$ & \multicolumn{2}{|c|}{$\begin{array}{c}\text { No }(\%) \text { of dataset } \\
A^{*}\end{array}$} & \multicolumn{2}{|c|}{$\begin{array}{c}\text { No (\%) of dataset } \\
\text { B十 }\end{array}$} & \multicolumn{2}{|c|}{$\begin{array}{c}\text { Dataset Aunivariate } \\
\text { analysis }\end{array}$} & \multicolumn{2}{|c|}{$\begin{array}{c}\text { Dataset A multivariate } \\
\text { random effects without } \\
\text { used mattress }\end{array}$} & \multicolumn{2}{|c|}{$\begin{array}{l}\text { Dataset A multivariate } \\
\text { random effects with } \\
\text { used mattress }\end{array}$} & \multicolumn{2}{|c|}{$\begin{array}{c}\text { Dataset B conditional } \\
\text { multivariatewith used } \\
\text { mattress }\end{array}$} \\
\hline & $\begin{array}{cc}\text { Cases } & \text { Controls } \\
(n=131) & (n=278)\end{array}$ & $\begin{array}{l}\text { Cases } \\
(\mathrm{n}=117)\end{array}$ & $\begin{array}{l}\text { Controls } \\
(n=265)\end{array}$ & $\begin{array}{l}\text { Cases } \\
(n=111)\end{array}$ & $\begin{array}{l}\text { Controls } \\
(\mathrm{n}=202)\end{array}$ & $\begin{array}{c}\text { OR } \\
(95 \% \mathrm{CI})\end{array}$ & $\begin{array}{c}\mathbf{P} \\
\text { value }\end{array}$ & $\begin{array}{c}\text { OR } \\
(95 \% \mathrm{CI})\end{array}$ & $\begin{array}{c}P \\
\\
\text { value }\end{array}$ & $\begin{array}{c}\mathrm{OR} \\
(95 \% \mathrm{Cl}) \\
\end{array}$ & $\begin{array}{c}P \\
\text { value }\end{array}$ & $\begin{array}{c}\mathrm{OR} \\
(95 \% \mathrm{Cl}) \\
\end{array}$ & $\begin{array}{c}P \\
\text { value }\end{array}$ \\
\hline \multicolumn{14}{|c|}{ Infant mattress new or used } \\
\hline \multicolumn{14}{|l|}{ Routine sleep: } \\
\hline New & & $\begin{array}{c}46 / 101 \\
(46)\end{array}$ & $\begin{array}{c}178 / 246 \\
(72)\end{array}$ & $\begin{array}{c}44 / 96 \\
(46)\end{array}$ & $\begin{array}{c}135 / 186 \\
(73)\end{array}$ & 1 & & & & 1 & & 1 & \\
\hline \multicolumn{14}{|c|}{ How many users: } \\
\hline 0 & & $\begin{array}{c}46 / 95 \\
(48)\end{array}$ & $\begin{array}{c}178 / 245 \\
(72)\end{array}$ & $\begin{array}{c}44 / 91 \\
(48) \\
\end{array}$ & $\begin{array}{c}135 / 186 \\
(72)\end{array}$ & 1 & & & & 1 & & & \\
\hline 1 & & $\begin{array}{l}31 / 95 \\
(33)\end{array}$ & $\begin{array}{c}48 / 245 \\
(20)\end{array}$ & $\begin{array}{c}29 / 91 \\
(32)\end{array}$ & $\begin{array}{c}37 / 186 \\
(20)\end{array}$ & $\begin{array}{c}2.50 \\
(1.38 \text { to } 4.52)\end{array}$ & 0.002 & & & $\begin{array}{c}2.58 \\
(1.17 \text { to } 5.70)\end{array}$ & 0.019 & & \\
\hline$\geqslant 2$ & & $\begin{array}{c}18 / 95 \\
(19)\end{array}$ & $\begin{array}{c}19 / 245 \\
(8)\end{array}$ & $\begin{array}{c}18 / 91 \\
(20)\end{array}$ & $\begin{array}{c}14 / 186 \\
(8)\end{array}$ & $\begin{array}{c}3.67 \\
\text { (1.68 to } 8.01)\end{array}$ & $<0.001$ & & & $\begin{array}{c}4.19 \\
(1.55 \text { to } 11.32)\end{array}$ & 0.005 & & \\
\hline New & & $\begin{array}{c}46 / 101 \\
(46)\end{array}$ & $\begin{array}{c}178 / 246 \\
(72)\end{array}$ & $\begin{array}{c}44 / 96 \\
(46)\end{array}$ & $\begin{array}{c}135 / 186 \\
(73)\end{array}$ & 1 & & & & 1 & & & \\
\hline $\begin{array}{l}\text { Used same } \\
\text { home }\end{array}$ & & $\begin{array}{c}17 / 101 \\
(17)\end{array}$ & $\begin{array}{c}42 / 246 \\
(17)\end{array}$ & $\begin{array}{l}15 / 96 \\
(16)\end{array}$ & $\begin{array}{c}34 / 186 \\
(18)\end{array}$ & $\begin{array}{c}1.50 \\
(0.75 \text { to } 3.00)\end{array}$ & 0.29 & & & $\begin{array}{c}1.64 \\
(0.64 \text { to } 4.20)\end{array}$ & 0.30 & & \\
\hline $\begin{array}{l}\text { Used other } \\
\text { home }\end{array}$ & & $\begin{array}{c}38 / 101 \\
(38)\end{array}$ & $\begin{array}{c}26 / 246 \\
(11)\end{array}$ & $\begin{array}{c}37 / 96 \\
(39)\end{array}$ & $\begin{array}{c}17 / 186 \\
(9)\end{array}$ & $\begin{array}{c}5.42 \\
(2.88 \text { to } 10.24)\end{array}$ & $<0.001$ & & & $\begin{array}{c}4.78 \\
(2.08 \text { to } 11.00)\end{array}$ & $<0.001$ & & \\
\hline \multicolumn{14}{|c|}{ Last sleep and not sharing: } \\
\hline New & & $\begin{array}{c}17 / 44 \\
(39)\end{array}$ & $\begin{array}{c}151 / 205 \\
(74)\end{array}$ & $\begin{array}{c}17 / 43 \\
(40)\end{array}$ & $\begin{array}{c}112 / 152 \\
(74)\end{array}$ & 1 & & & & 1 & & & \\
\hline Used & & $\begin{array}{c}27 / 44 \\
(61)\end{array}$ & $\begin{array}{c}54 / 205 \\
(26)\end{array}$ & $\begin{array}{c}26 / 43 \\
(60)\end{array}$ & $\begin{array}{c}40 / 152 \\
(26)\end{array}$ & $\begin{array}{c}4.44 \\
(2.14 \text { to } 9.30)\end{array}$ & $<0.001$ & & & $\begin{array}{c}6.10 \\
(2.31 \text { to } 16.12)\end{array}$ & $<0.001$ & & \\
\hline
\end{tabular}

${ }^{*}$ Routine cosleepers excluded.

†Includes only cases with matched controls and no routine cosleepers.

Odds ratios continuous/multicategory: maternal age per year, infant age per day, deprivation category per single level, parity per extra infant, share yes/no.

infant mattress, we attempted to force in a further strong variable, mother not in paid employment before baby's birth. This did not change the significance of used infant mattress, which argues against the presence of residual socioeconomic confounding.

Other obvious confounding items such as parity and sharing beds on the night of death were in the multivariate model. Restriction of the dataset to look at used infant mattress during last sleep in those not sharing during last sleep removed any bed sharing effect, but the association remained.

\section{What is already known about this topic}

The major risk factors for sudden infant death syndrome are sleeping prone and parental smoking

One study has suggested that the syndrome is associated with sleeping on an infant mattress previously used by another child

\section{What this study adds}

New case-control data show that the association between a previously used infant mattress and sudden infant death syndrome is valid

When source of used mattress is categorised, the association is significant only if the mattress is from another home

Insufficient evidence is available to judge whether this is a cause and effect relation

\section{Cause and effect}

The association between sudden infant death syndrome and used infant mattresses is relatively strong (odds ratio 3), and the time sequence of use of mattress and death is compatible. In addition, toxigenic bacteria that have been implicated in sudden infant death syndrome ${ }^{56}$ do reside in used infant mattresses. ${ }^{7}$ However, we found no dose-response effect and the only other study with similar information did not show a link between old infant mattresses and the sudden infant death syndrome. ${ }^{8}$

\section{Conclusions}

As our findings are relatively robust over a range of statistical approaches, we conclude that there is a valid statistical association between sudden infant death syndrome and use of an infant mattress previously used by another child, particularly if from another home. We are therefore able to reject the null hypothesis. There remains insufficient evidence to establish a cause and effect relation.

Contributors: The study was the idea of $\mathrm{HB}$ and $\mathrm{AG}$ who applied for ethics approval, secured funding, constructed the questionnaire, coded the data, and helped write the paper. DT helped construct the questionnaire, code the data, worked closely with RE during the analysis, and wrote the paper. RE analysed the data and helped write the paper. DT is guarantor.

Funding: Scottish Cot Death Trust.

Competing interests: None declared.

Brooke H, Gibson A, Tappin D, Brown H. Case-control study of sudden infant death syndrome in Scotland 1992-5. BMJ 1997:314:1516-20.

2 Gibson AAM. The use of a standard SIDS post mortem protocol in Scotland: two years' audit. In: Third SIDS international conference, Stavanger.Norway, 1994. Oslo: Norwegian SIDS Society, 1994.

3 Carstairs V, Morris R. Deprivation and health in Scotland. Aberdeen: Aberdeen University Press, 1991. 
4 STATA. Stata7 User's Guide, Release 7. College Station, TX: Stata Press, 2001.

Blackwell CC, Saadi AT, Raza MW, Stewart J, Weir DM. Susceptibility to infection in relation to SIDS. J Clin Pathol 1992; 44 (suppl I): 20-4.

6 Blackwell CC, MacKenzie DA, James VS, Elton RA, Zorgani AA, Weir DM, Busuttil A. Toxigenic bacteria and sudden infant death syndrome (SIDS): nasopharyngeal flora during the first year of life. FEMS Immunol Med Microbiol 1999;25:199-206.
7 Jenkins R. Cot mattresses: a reservoir of toxigenic bacteria. Proceedings of the Scottish Cot Death Trust grant holders meeting. March 2002.Glasgow: SCDT, 2002.

8 Fleming P, Bacon C, Blair P, Berry PJ, eds. Sudden unexpected death in infancy. CESDI SUDI studies 1993-1996. London: Stationery Office, 2000:50-1.

(Accepted 23 July 2002) 